\title{
Reconstruction of the larynx with unipedicled sternohyoid myofascial flap following open extended vertical partial laryngectomy
}

\author{
Ramil K. Yagudin ${ }^{1}$, Kamil. F. Yagudin ${ }^{2}$
}

\author{
${ }^{1}$ Department of Otorhinolaryngology, Kazan State Medical Academy, Kazan, Russia \\ ${ }^{2}$ Department of Otolaryngology, Kazan 8th Polyclinic, Kazan, Russia
}

Received: 06 September 2018

Accepted: 14 Novemober 2018

\section{*Correspondence:}

Dr. Ramil K. Yagudin,

E-mail: ramil.k.yagudin@gmail.com

Copyright: (C) the author(s), publisher and licensee Medip Academy. This is an open-access article distributed under the terms of the Creative Commons Attribution Non-Commercial License, which permits unrestricted non-commercial use, distribution, and reproduction in any medium, provided the original work is properly cited.

\begin{abstract}
The extent of resection during open vertical partial laryngectomy may vary considerably and even an experienced surgeon may encounter difficulties in reconstructing the larynx to preserve laryngeal function and to prevent stenosis. Different local flaps were proposed for reconstruction; however, the ideal technique is still under development. A simple modification to the technique originally described by Calcaterra (1983) is presented. The unipedicled sternohyoid myofascial flap consists of the unsplit sternohyoid muscle with all three overlying fasciae harvested as a whole. The flap is rotated $90^{\circ}$ and horizontally sutured in position to supply the necessary bulk to the entire neocord to produce a functional voice. Completely filling the mucosal defect USMF-flap allows laryngeal structures to maintain their native position and retain protective function of the larynx. The thick multilayered fascia covering facilitates rapid epithelization and prevents excessive granulation and secondary stenosis. The technique is indicated in glottic cancer patients treated by open extended vertical partial laryngectomy when the laryngeal mucosa defect is too extensive for primary closure and poses significant risk of developing postoperative stenosis.
\end{abstract}

Keywords: Glottic cancer, Open extended partial laryngectomy, Flap reconstruction

\section{INTRODUCTION}

The extent of laryngeal structure resection during open vertical partial laryngectomy may vary considerably and successful reconstruction of the extended mucosal defect can be a challenge even for an experienced surgeon. If the resultant soft tissue defect is over $2.5 \mathrm{~cm}$ in vertical dimension, the simple reconstruction with direct suturing is often impossible. The remaining remnants of subglottic mucosa are tightly adherent to the cartilage and cannot be advanced up enough. Excessive pulling of the more loosely attached supraglottic mucosa down entails displacement of the aryepiglottic fold inwards and the epiglottis backwards that can subsequently cause permanent narrowing of the airway and affect postoperative voice quality. Moreover, it may potentially result in aspiration. Further follow-up as well as early detection of local recurrence may be complicated. When large amount of the contralateral vocal cord is removed, then reconstruction with epiglottopexy may suffice. The epiglottis is released and sutured to the cricoid, providing epithelial lining and cartilage support. Unfortunately, the epiglottis is often poorly mobile after previous radiation or its petiole may be involved by tumor that makes epiglottopexy unsuitable.

Although the large mucosal defect can be left uncovered, it inevitably results in excessive granuloma and scar formation. The healing is delayed and laryngeal stenosis may develop especially if extended partial laryngectomy is performed as a salvage surgery after chemoradiation therapy. The detection of tumor recurrence behind fibrous tissue may be similarly difficult. 
Laryngeal reconstruction using local flaps avoids risk of direct mucosal closure with undue tension. It allows resurfacing large raw areas of cartilage and prevents chondritis and cartilage necrosis. Filling the defect with vascularized tissue having sufficient bulk to replace the excised laryngeal structures provides restoration of glottic competence and permits reconstructed vocal fold to produce a functional voice.

Here, a simple modification to the technique originally described by Calcaterra is presented. ${ }^{1}$ The unipedicled sternohyoid myofascial flap (USMF-flap) consists of the "unsplit" sternohyoid with not muscular, as opposed to Calcaterra flap, but all three overlying fasciae, namely superficial, investing and muscular, harvested as a whole. This simple modification facilitates the technical aspects of the flap harvest, provides additional tissue bulk, the multilayered fascia sheet offers greater resistance to infection. In contrast to Calcaterra flap, the USMF-flap is rotated $90^{\circ}$ and horizontally sutured in position to supply the necessary bulk to the entire "neocord".

\section{TECHNIQUE}

Tracheostomy is performed. After the collar incision a cervical skin flap is elevated in the subcutaneous plane. The strap muscles are separated in the midline and the thyroid cartilage is exposed. Then vertical partial laryngectomy is undertaken. The thyroid cartilage is cut vertically on both sides of larynx to include the central segment of cartilage adjacent to the anterior commissure in the surgical specimen. The cartilage cuts are designed depending on the tumor extension and anterior commissure invasion; however it is important to avoid extensive cartilage resection to success in reconstruction with the USMF-flap. At least half of the thyroid cartilage on the involved side and the two-thirds on the contralateral side are preserved to retain rigidity of reconstructed larynx. If cartilage preservation is not suitable then other glottic reconstruction techniques or total laryngectomy should be considered. The laryngeal lumen is entered and then tumor resection is carried out. Following excision the size of the defect is assessed and if it is too large to perform a direct mucosal approximation, the ipsilateral USMF-flap is planned.

The unipedicled sternohyoid myofascial flap consists of the sternohyoid muscle and the three overlying fasciae harvested as a whole. The flap may be superiorly or inferiorly based depending on patient anatomy and surgeon preference. To raise the flap the skin is undermined in the subcutaneous plane. The sternohyoid is separated from the underlying strap muscles and the rectangular flap is fashioned from medial edge of sternohyoid adjacent to the defect (Figure 1).

It is essential to adjust the width of the USMF-flap according to the axial size of the laryngeal defect so that after the flap is rotated $90^{\circ}$ and transferred to the recipient site tensionless closure of the mucosal gap can be achieved without distortion of the native topography of the laryngeal inlet. The flap is sutured to the mucosal margins of resection with absorbable sutures with the fascia facing the airway (Figure 2).

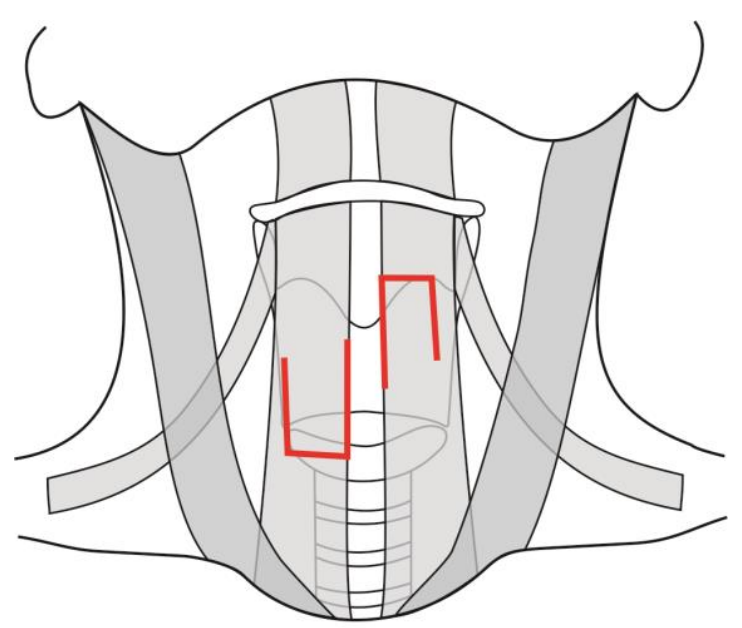

Figure 1: The USMF-flap can be superiorly or inferiorly based depending on the neck anatomy.

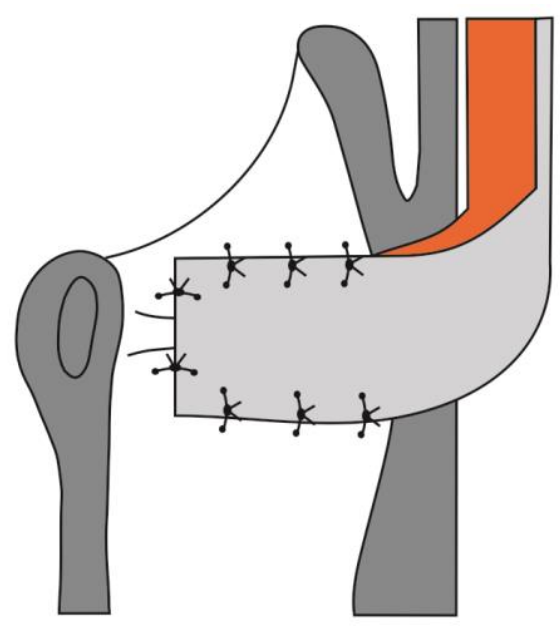

Figure 2: The position of the USMF-flap in the larynx.

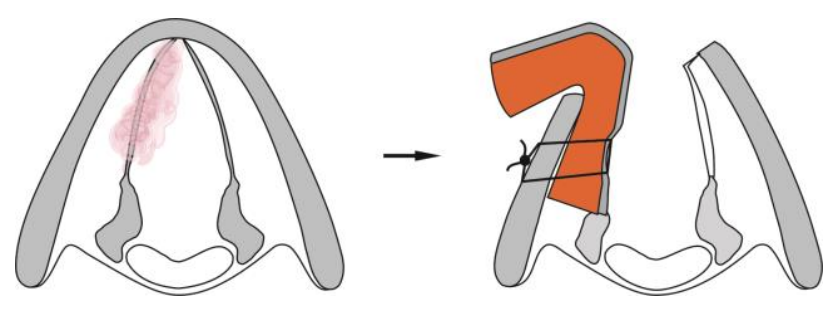

Figure 3: Vertical partial laryngectomy with the

USMF flap reconstruction. The top-view scheme.

Note partial preservation of thyroid cartilage to success in reconstruction.

One or two through-and-through absorbable mattress sutures can be applied and tied on the external surface of thyroid ala to ensure close adaptation of the flap to the underlying cartilage (Figure 3). 
Additionally, the larynx may be temporally packed with ribbon gauze. This packing of larynx is intended to prevent postoperative bleeding and to allow feeding by mouth to start within 24 hours after surgery. The gauze packing may be in place for 1-4 days and during this period the patient is forced to breathe through the tracheostomy tube. Then the pack is removed and the next day the patient may be decannulated.

\section{DISCUSSION}

Different local flaps were proposed for glottic reconstruction after creating large mucosal defects. Pressman (1954) was the first who used the whole strap muscles as bipedicled muscle flap. He sutured the external perichondrium of the larynx to the strap muscles to form an internal lining of newly constructed laryngeal wall and transplanted the removed thyroid ala in the pocket between the muscle layers to provide rigidity. ${ }^{2}$ The disadvantage of the technique was a breathy voice and collapsibility of the airway requiring laryngeal stenting and long-term tracheostomy. A few other bipedicled muscle flaps were proposed subsequently however, they did not produced the desired result either, because created tissue bulk predominantly in the anterior glottis, whereas it was also necessary in the posterior aspect to prevent glottic incompetence and to improve voice. $^{3,4}$

In 1969, Ogura and Biller proposed a unipedicled muscle flap from the sternohyoid to obtain bulk in the posterior aspect of glottis while the mucosal covering was obtained with rotation of the aryepiglottic fold, the thyroid ala was still completely removed. ${ }^{5}$ Calcaterra (1983) introduced a combined myofascial flap, where the muscular fascia covering was used instead of perichondrium to avoid excessive granulation and secondary stenosis. ${ }^{1}$ Calcaterra flap was a superiorly-based, single-pedicled flap fashioned from the posterolateral edge of sternohyoid, yet it was vertically oriented like bipedicled flaps. Inclusion of the muscular fascia in the flap permitted ease of approximation to the remaining laryngeal mucosa and subsequent rapid epithelization. Because of low metabolic activity, the fascia was resistant to saliva, infection and irradiation. Still, complete removal of thyroid ala was advocated and the surgeons had to split the sternohyoid in the vertical plane employing a part of the strap muscle to provide structural support to the laryngeal lumen. Consequently, the laryngeal stenosis may develop due to potential collapsibility of the reconstructed wall. ${ }^{1}$

Elo (2000) proposed a wide laterally pedicled fascia-only flap to cover glottic defect; the role of the sternohyoid muscle was just to contribute support to partly preserved laryngeal framework. ${ }^{6}$ A major shortcoming of sole fascia as a reconstructive material was lack of bulk with resultant incomplete glottic closure and breathy voice.

A few other reconstruction techniques to fill the extended vertical partial laryngectomy defect and to prevent laryngeal stenosis have been reported such as the thyroid gland flap, the radial forearm flap with a skin paddle, the temporoparietal free flap combined with cartilage and mucosal grafts, the combined hyoid bone flap, the neovascularised tracheal autograft. ${ }^{7-11}$ However, most of them are rather difficult to perform and none has been mentioned yet as the ideal technique.

The USMF-flap is a modification to the technique originally described by Calcaterra and consists of the "unsplit" sternohyoid with not muscular, as opposed to Calcaterra flap, but all three overlying fasciae, harvested as a whole. In contrast to Calcaterra flap, the USMF-flap is rotated $90^{\circ}$ and horizontally sutured in position to supply the necessary bulk to the entire "neocord". USMF-flap when completely filling the mucosal defect allows laryngeal structures to maintain their native position and retain protective function of the larynx, and therefore, normal swallowing is resumed within one to two days after the removal of the laryngeal packing and nasogastric feeding is never necessary.

The thick multilayered fascia covering enables a meticulous approximation of the flap edges to the remaining remnants of laryngeal mucosa and also facilitates rapid epithelization of the flap. Similar to other fascia-containing flaps excessive granulation and secondary stenosis is avoided. ${ }^{6}$ Postoperative radiotherapy, if received, can also have a positive impact on laryngeal lumen due to inhibition of granulation tissue growth. The muscle placed transversally in the position provides excellent soft tissue bulk to produce a functional voice. Finally, the entire flap reliably protects the thyroid cartilage against postoperative infection.

Unlike the many laryngeal reconstruction techniques where flaps are elevated before the surgeon can enter the larynx and assess the tumor margins, the USMF-flap is raised after the tumor has been completely removed. ${ }^{6,12,13}$ Therefore, the flap can be easily tailored to the size of extended vertical partial laryngectomy defect. Because of close proximity of the donor site to the laryngeal defect a short pedicle of the flap ensures reliable blood supply, and subsequently it may decrease the chance for flap atrophy and glottic insufficiency. Similar to other strap muscle flaps it is harvested with minimal donor site morbidity.

To reconstruct the larynx following open extended vertical partial laryngectomy with USMF-flap, it is essential to preserve enough cartilage framework, otherwise the larynx may be at risk of postoperative stenosis. Partial preservation of ipsilateral thyroid cartilage ala provides advantage of maintaining laryngeal wall rigidity and greatly simplifies the procedure. Preoperative assessment of tumor invasion of the laryngeal framework is difficult; however clinically early-stage glottic cancer that does not impair vocal fold mobility is unlikely to infiltrate thyroid cartilage. The only exception is when the tumors involve anterior 
commissure, but even in these cases incidence of cartilage invasion (mostly microinvasion) is not high. ${ }^{14}$ Ulusan et al found cartilage invasion rate of $5.4 \%$ in early tumors limited to the glottis with anterior commissure involvement and significantly higher cartilage invasion rate of $32 \%$ when early glottic cancer invaded both anterior commissure and supraglottis. ${ }^{15}$ Nevertheless, cartilage invasion was only observed in the thyroid angle and never affected the lateral or posterior of the thyroid ala. Therefore, wide or complete resection of the thyroid cartilage as has been previously advocated by many authors does not seem necessary in early stage cancers and may be limited to not more than a half of the thyroid lamina on the affected side and the anterior one third on the opposite side. If partial preservation of the cartilage framework is utilized, a greater amount of endolaryngeal structures may be removed without risk of postoperative stenosis and the need to transpose or implant supporting structures.

In the era of worldwide use of transoral laser microsurgery for early laryngeal cancer open extended partial laryngectomy still may have advantage of perfect tumor visualization, wide cartilage resection and samestage reconstruction. ${ }^{16,17}$ Candidates for the USMF-flap reconstruction following open vertical partial laryngectomy are a selected group of T2 glottic cancer patients with anterior commissure and supraglottis involvement when the resulted operative defect is too large to be closed by direct suturing and when the partial framework preservation can be undertaken without compromising local control. The obvious contraindication is the presence of metastasis in the regional lymph nodes.

Over the last 25 years, the unipedicled sternohyoid myofascial flap was used for reconstruction in 76 out of 196 patients with T2 glottic cancers treated by open vertical partial laryngectomy. No flap-related complications occurred neither intraoperative nor the cases of postoperative flap necrosis. All the patients with the USMF-flap reconstruction were successfully decannulated after surgery and only one of them later developed laryngeal stenosis related to the excessive cartilage resection.

\section{CONCLUSION}

The USMF-flap is a very simple to perform, reliable and not time-consuming technique for defect reconstruction following open extended vertical partial laryngectomy. It is an effective alternative to the variety of more complicated reconstructions in a selected group of clinically $\mathrm{T} 2$ glottic cancer patients in which the partial framework preservation is possible without compromising oncologic outcomes.

Funding: No funding sources Conflict of interest: None declared Ethical approval: Not required

\section{REFERENCES}

1. Calcaterra TC. Sternohyoid myofascial flap reconstruction of the larynx for vertical partial laryngectomy. Laryngoscope 1983;93:422-4.

2. Pressman JJ. Cancer of the larynx; laryngoplasty to avoid laryngectomy. AMA Arch Otolaryngol 1954;59:395-412.

3. Maran AG, Hast MH, Leonard JR. Reconstructive surgery for improved glottic closure and voice following partial laryngectomy: an experimental study. Laryngoscope 1968;78:1916-36.

4. Bailey BJ. Partial laryngectomy and laryngoplasty. Laryngoscope 1971;81:1742-71.

5. Ogura JH, Biller HF. Glottic reconstruction following extended frontolateral hemilaryngectomy. Laryngoscope 1969;79:2181-4.

6. Elo J, Horvath E, Kesmarszky R. A new method for reconstruction of the larynx after vertical partial resections. European archives of oto-rhinolaryngology 2000;257:212-5.

7. Ikeda A, Okamoto H, Tsuchiya Y, Nishimura K, Ueda H, Ogawa T. Case of partial vertical laryngectomy with vocal cord reconstruction using a thyroid gland flap. Head Neck 2014;36:E73-7.

8. Delaere P, Vander Poorten V, Vranckx J, Hierner R. Laryngeal repair after resection of advanced cancer: an optimal reconstructive protocol. Eur Arch Otorhinolaryngol 2005;262:910-6.

9. Gilbert RW, Goldstein DP, Guillemaud JP, Patel RS, Higgins KM, Enepekides DJ. Vertical partial laryngectomy with temporoparietal free flap reconstruction for recurrent laryngeal squamous cell carcinoma: technique and long-term outcomes. Arch Otolaryngol Head Neck Surg 2012;138:484-91.

10. Ouyang D, Liu TR, Liu XW, Chen YF, Wang J, Su $\mathrm{X}$, et al. Combined hyoid bone flap in laryngeal reconstruction after extensive partial laryngectomy for laryngeal cancer. European archives of otorhino-laryngology 2013;270:1455-62.

11. Leong SC, Upile N, Lau A, Lancaster J, Praveen P, Rogers SN, et al. Extended vertical hemilaryngectomy and reconstruction with a neovascularised tracheal autograft for advanced unilateral laryngeal tumours: a learning curve. European archives of oto-rhino-laryngology 2014;271:1729-35.

12. Dursun G, Ozgursoy OB. Laryngeal reconstruction by platysma myofascial flap after vertical partial laryngectomy. Head \& neck 2005;27:762-70.

13. Jurlina M, Matulić Z, Prstačić R, Janjanin S. Composite platysmofascial flap in reconstruction following partial vertical laryngeal resections. Laryngoscope. 2011;121:1899-901.

14. Hartl DM, Landry G, Hans S, Marandas P, Brasnu DF. Organ preservation surgery for laryngeal squamous cell carcinoma: low incidence of thyroid cartilage invasion. Laryngoscope 2010;120:1173-6.

15. Ulusan M, Unsaler S, Basaran B, Yilmazbayhan D, Aslan I. The incidence of thyroid cartilage invasion 
through the anterior commissure in clinically earlystaged laryngeal cancer. European Archives of Otorhino-laryngol. 2016;273:447-53.

16. Mortuaire G, Chevalier D, Mouawad F. Open partial laryngectomy after failure of (chemo) radiation: indications, oncologic and functional outcomes. Curr Opin Otolaryngol Head Neck Surg. 2017;25:159-62.

17. Wolber P, Schwarz D, Stange T, Ortmann M, Balk M, Anagiotos A, et al. Surgical Treatment for Early
Stage Glottic Carcinoma with Involvement of the Anterior Commissure. Otolaryngol Head Neck Surg. 2018;158:295-302.

Cite this article as: Yagudin RK, Yagudin KF. Reconstruction of the larynx with unipedicled sternohyoid myofascial flap following open extended vertical partial laryngectomy. Int J Otorhinolaryngol Head Neck Surg 2019;5:231-5. 